\title{
The Impact of Enteric Contrast on Radiologist Confidence in Intravenously Enhanced MDCT of the Abdomen and Pelvis: A Randomized Controlled Trial
}

\author{
Christopher M. Garcia1, Steven Boe', Bret Coughlin'1,2, David M. 0'Sullivan³, \\ Douglas Moote',2, Michael T. 0’Loughlin',2, Devika Jajoo ${ }^{1,2}$, Steve Lee1,2 \\ ${ }^{1}$ Department of Radiology, Hartford Hospital, Hartford, USA \\ 2 Jefferson Radiology PC, East Hartford, USA \\ ${ }^{3}$ Department of Research, Hartford Hospital, Hartford, USA \\ Email: Christopher.michael.garcia@gmail.com
}

Received 29 March 2014; revised 29 April 2014; accepted 4 May 2014

Copyright (C) 2014 by authors and Scientific Research Publishing Inc.

This work is licensed under the Creative Commons Attribution International License (CC BY). http://creativecommons.org/licenses/by/4.0/

(c) (i) Open Access

\begin{abstract}
After Institutional Review Board approval, 109 patients $\geq 18$ years old undergoing intravenously enhanced Computed Tomography (CT) of the abdomen and pelvis were prospectively enrolled and randomized into two arms. Patients with a history of inflammatory bowel disease, Crohn disease and ulcerative colitis were excluded. The control arm received routine enteric contrast and intravenous contrast. The experimental arm did not receive oral contrast, but received the standard dose of intravenous (IV) contrast. One of four experienced body radiologists reviewed the studies and rated his/her confidence on a scale of 1 (no confidence) to 10 (all confident findings have been made). Confidence levels were compared between groups. The 109 subjects were distributed 54 $(49.5 \%)$ in the control arm and $55(50.5 \%)$ in the experimental arm. The average confidence level in the control arm was $8.7 \pm 0.9$ versus $8.4 \pm 1.1$ in the experimental arm, which is not statistically significant ( $p$ value $=0.09$ ). Among radiologists, the average confidence levels ranged from $6-10$ in the control arm and $7-10$ in the experimental arm. No examinations were repeated for technical considerations in either group. No studies were repeated in the experimental group in order to administer oral contrast. When each radiologist was compared to the others, there were no differences in confidence level between pairs in the control arm; however, there were statistically significant differences in confidence level among three pairs of radiologists for the experimental arm. There was no statistically significant difference in radiologist confidence level between intravenously enhanced abdomen and pelvis CTs with enteric contrast and without enteric contrast.
\end{abstract}




\section{Keywords}

\section{Enteric Contrast, Outpatient CT, Confidence, Oral Contrast}

\section{Introduction}

Although protocols vary by institution, equipment, setting and clinical question, the default protocol in our practice for outpatient contrast-enhanced abdominal CT scans includes both enteric and IV contrast administration. Recently, the justification for enteric contrast in the emergency department has been questioned in multiple reports. Studies demonstrate the non-superiority of enteric contrast studies in diagnosing appendicitis [1]-[4] and traumatic bowel injury [5]. The majority of those studies focus on appendicitis, probably due to its clear surgical endpoint and relatively high prevalence. Other studies continue to demonstrate that regardless of the presence of enteric, intravenous, or even rectal contrast, multidetector computed tomography (MDCT) is highly sensitive and specific in diagnosing acute appendicitis [6] [7] and other acute processes [8].

The routine use of enteric contrast in the outpatient setting has multiple direct and indirect effects, including increased cost, decreased practice efficiency, patient inconvenience and sometimes discomfort. Enteric contrast in oncology patients has been associated with lower patient satisfaction scores [9]. Although the vast majority of research in this area is performed in the emergency setting, the advantages of withholding enteric contrast in the emergency department may translate to routine intravenously enhanced MDCT of the abdomen and pelvis in the outpatient setting. Our purpose in this randomized controlled trial is to evaluate the impact of enteric contrast in intravenously enhanced MDCT of the abdomen and pelvis on radiologist confidence in the outpatient setting.

\section{Materials and Methods}

\subsection{Study Design}

The trial was conducted following IRB approval and registration with a national database (www.clinicaltrials.gov, NCT01655550). Following informed consent, 109 patients $\geq 18$ years old undergoing intravenously contrast-enhanced CT of the abdomen and pelvis were prospectively enrolled and randomized into two arms in a one-to-one fashion between April 2012 and May 2013, inclusive. The control arm received routine enteric and IV contrast. The experimental arm did not receive enteric contrast, but received the standard dose of IV contrast. All patients ingested 6 ounces of water immediately prior to the scan. One of four experienced body radiologists with 13, 16, 16 and 24 years of practice beyond training was assigned, based on availability, to review each case and rate his/her confidence on a scale of 1 (no confidence) to 10 (confident all findings have been made). Interpreting radiologists underwent a one-hour training session in selecting the appropriate confidence level, where pilot cases were reviewed and scored. Axial images as well as coronal and sagittal reconstructions were provided for interpretation in both groups. See Figure 1 for the ten point scale of confidence levels. Briefly, a confidence level of 10 corresponded to "Excellent study. Confident all findings have been made" and a level of 1 corresponded to "Non diagnostic study. Study needs to be repeated". A level of 5 corresponded to "Somewhat limited study. May have missed a finding". Confidence levels were compared between groups.

Patients with a history of inflammatory bowel disease, Crohn disease and ulcerative colitis were excluded. Other exclusion criteria were allergy to IV contrast, pregnancy, and unwilling or unable to give informed consent. Patients with specific requisitions for CT enterography were excluded. Patient age, height, weight and body mass index were obtained prospectively.

\subsection{CT Technique}

Computed tomography scans were performed in two outpatient radiology offices. CT scans were performed on a GE VCT Light Speed 64 slice MDCT scanner with a 70-second scan delay after intravenous administration of $100 \mathrm{~mL}$ of Omnipaque $300^{\mathrm{TM}}$ (GE Healthcare, Waukesha, WI) at a rate of $3 \mathrm{ml}$ per second. Auto milliampere dose modulation was used, with eye, thyroid and breast bismuth shields. 40\% Adaptive Statistical Iterative Reconstruction was used. Subjects in the control arm ingested $450 \mathrm{~mL}$ of Readicat $\mathrm{II}^{\mathbb{B}}$ Barium Sulfate suspension $2.1 \% \mathrm{w} / \mathrm{v}, 2.0 \% \mathrm{w} / \mathrm{w}(\mathrm{E}-\mathrm{Z}-\mathrm{Em}$, Lake Success, NY) one hour before their scheduled appointment time. 


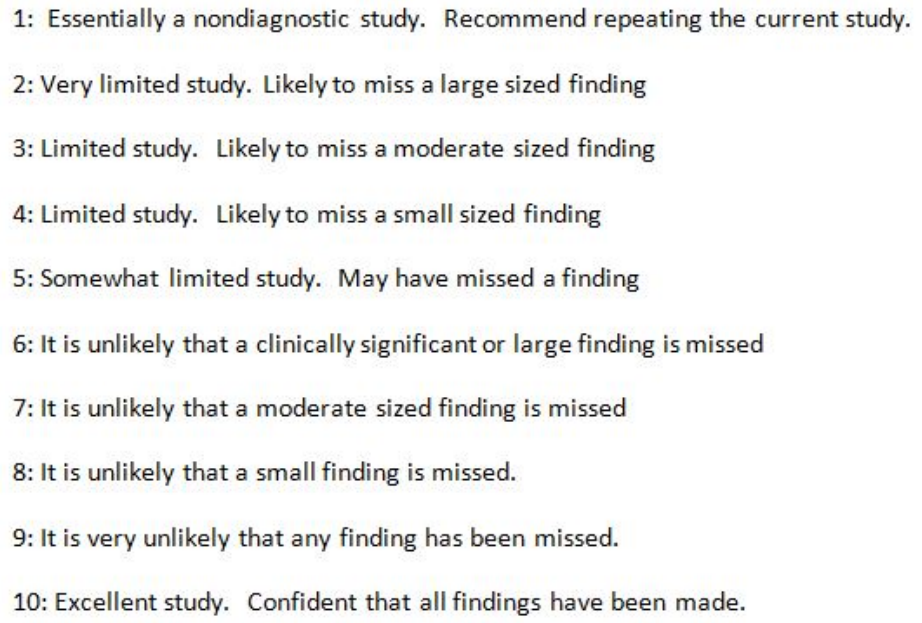

Figure 1. Confidence level worksheet used by radiologist.

\subsection{Data Analysis}

The data analyst was blinded with respect to patient allocation. The nature of enteric contrast does not allow for blinding of the interpreting radiologists. Continuous data were assessed for normality and found to be normally distributed. Inferential statistics comprised chi-square tests for categorical data and Student's t-tests for twogroup comparisons. One-way analysis of variance with post hoc Scheffé's multiple range tests were used for comparisons involving more than two groups. Statistical analyses were conducted with IBM SPSS v. 21 (IBM, Armonk, NY 2013), using an a priori alpha level of 0.05 such that all results yielding $p<0.05$ were deemed statistically significant.

\section{Results}

The 109 subjects were distributed $54(49.5 \%)$ in the control arm and $55(50.5 \%)$ in the experimental arm. At baseline, there were no differences between groups with respect to age or BMI (Table 1). The most common indication for contrast enhanced CT scans in both groups was cancer staging, 36/56 (64\%) in the control group and 38/55 (69\%) in the experimental group. Abdominal pain was the next most likely indication $8 / 56(15 \%)$ in the control group and 10/55 (18\%) in the experimental group (Table 2).

Among radiologists, the average confidence levels ranged from $6-10$ in the control arm and $7-10$ in the experimental arm. The average confidence level ( $+/-$ standard deviation) in the control arm was $8.7 \pm 0.9$ versus $8.4 \pm 1.1$ in the experimental arm, with a p value of 0.09 . One reader had a statistically significant difference in confidence level; average confidence level of 8.6 in the enteric contrast group versus 7.7 in the no enteric contrast group, with a $\mathrm{p}$ value of 0.005 . Other readers did not have a statistically significant difference (Table 3 ).

There was no overall difference in confidence level by reader in the control group (ANOVA, $p=0.923$ ), with all inter-reader differences (i.e., 1 vs. 2, 1 vs. 3, etc.) $\mathrm{p}>0.93$. However, there was a significant difference in confidence level by reader in the experimental group (ANOVA, $\mathrm{p}<0.001$ ), with individual differences seen between readers 1 and 3 (Scheffé's, $p=0.011$ ), readers 1 and 4 (Scheffé's, $p<0.001$ ), and readers 2 and 4 (Scheffé's, $\mathrm{p}=0.001$ ).

A post hoc power analysis of our data revealed that group sample sizes of 54 and 55 achieved $40 \%$ power to detect a difference of 0.3 between the null hypothesis that both group means were 8.7 and the alternative hypothesis that the mean of group 2 was 8.4 , with known group standard deviations of 0.9 and 1.1 , using a two-sided t-test with a significance level (alpha) of 0.05 .

No examinations were repeated for technical consideration in either group. In $69 \%$ of studies in the experimental group, the radiologist felt that the addition of enteric contrast would not have impacted the confidence level. There were 9 studies in each group where follow-up examinations were recommended. No follow up recommendations were made due to inadequate bowel evaluation or to follow up abnormalities in the small or large bowel. 
Table 1. Subject data in the control group and the experimental group.

\begin{tabular}{cccc}
\hline & Enteric contrast & No enteric contrast & $\mathrm{p}$ value (Student t-test) \\
\hline Subject n $(\%)$ & $54(49.5 \%)$ & $55(50.5 \%)$ & \\
Age, years $($ mean $\pm \mathrm{SD})$ & $60.4 \pm 13.2$ & $61.2 \pm 11.2$ & 0.743 \\
Male/Female & $18 / 36$ & $26 / 29$ & 0.138 \\
$\mathrm{BMI}, \mathrm{kg} / \mathrm{m}^{2}($ mean $\pm \mathrm{SD})$ & $28.9 \pm 5.9$ & $28.5 \pm 6.1$ & 0.764 \\
\hline
\end{tabular}

Table 2. Total number of examinations by indication in each arm.

\begin{tabular}{ccc}
\hline Indication & Enteric Contrast $(\mathrm{n}=56)$ & No Enteric Contrast $(\mathrm{n}=55)$ \\
\hline Cancer Staging & $36(64 \%)$ & $38(69 \%)$ \\
Abdominal Pain & $8(14 \%)$ & $10(18 \%)$ \\
Other & $12(21 \%)$ & $7(13 \%)$ \\
\hline
\end{tabular}

Table 3. Average confidence levels, standard deviation and p values for both arms with subgroup analysis.

\begin{tabular}{|c|c|c|c|c|}
\hline \multirow{2}{*}{ Radiologist } & \multicolumn{3}{|c|}{ Confidence level } & \multirow[b]{2}{*}{$\mathrm{p}$ value (Student $\mathrm{t}$-test) } \\
\hline & Group & $\mathrm{N}$ & Mean \pm SD & \\
\hline \multirow[t]{2}{*}{ Total } & Enteric Contrast & 54 & $8.7 \pm 0.9$ & 0.090 \\
\hline & No Enteric Contrast & 55 & $8.4 \pm 1.1$ & \\
\hline \multirow[t]{2}{*}{ Radiologist 1} & Enteric Contrast & 8 & $8.6 \pm 0.7$ & 0.005 \\
\hline & No Enteric Contrast & 18 & $7.7 \pm 0.6$ & \\
\hline \multirow[t]{2}{*}{ Radiologist 2} & Enteric Contrast & 20 & $8.7 \pm 0.8$ & 0.14 \\
\hline & No Enteric Contrast & 10 & $7.8 \pm 1.0$ & \\
\hline \multirow[t]{2}{*}{ Radiologist 3} & Enteric Contrast & 13 & $8.9 \pm 1.0$ & 0.88 \\
\hline & No Enteric Contrast & 14 & $8.8 \pm 1.2$ & \\
\hline \multirow[t]{2}{*}{ Radiologist 4} & Enteric Contrast & 13 & $8.8 \pm 1.2$ & 0.13 \\
\hline & No Enteric Contrast & 13 & $9.4 \pm 0.7$ & \\
\hline
\end{tabular}

\section{Discussion}

Barium and water soluble enteric contrast agents have been in use for approximately a century, first in fluoroscopic examinations and now in CT [10]. Historically, enteric contrast was unquestionably necessary due to slow acquisition, bowel motion and relatively decreased contrast enhancement. Newer techniques and faster acquisitions have markedly improved temporal and spatial resolution of CT scans of the abdomen and pelvis, and therefore the need for enteric contrast should be reassessed. The use of enteric contrast in abdomen and pelvis CT has been both defended and called into question in recent years in multiple applications. Results from our study suggest that the addition of enteric contrast does not significantly add to the diagnostic confidence of the interpreting radiologist with intravenously enhanced studies. However, when readers were stratified individually, one reader did have a statistically significant difference in confidence level. Another reader had a higher confidence level in the group without enteric contrast, though the results are not statistically significant $(p=0.125)$. This suggests that personal preference and comfort level with/without routine enteric contrast may impact the confidence level of some radiologists. Examples of scored examinations at both extremes can be found in Figure 2 and Figure 3.

Although the aim of this study was to assess the role enteric contrast plays in the experience of the interpreting radiologist, one must also consider its impact on the patient. Patients need to pick up the bottle of contrast 


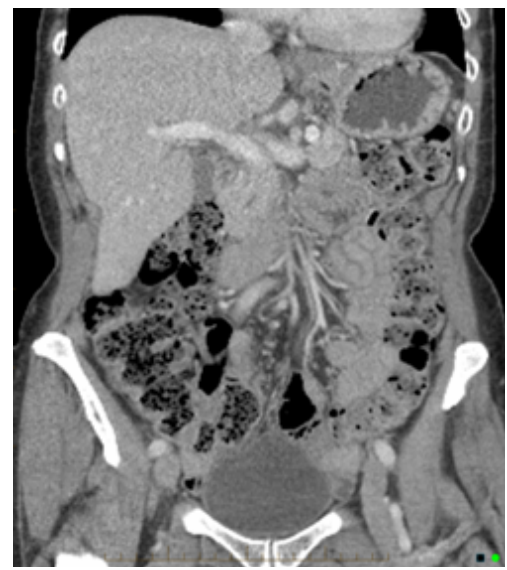

(a)

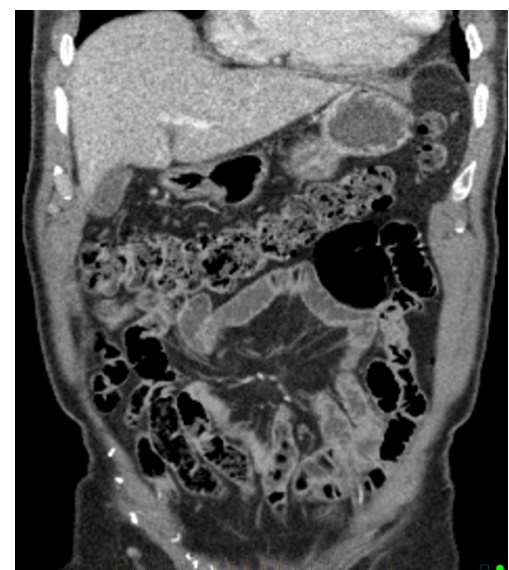

(b)

Figure 2. Coronal images without enteric contrast. (a) One of the lowest confidence levels, 7. Lack of intra-abdominal fat limits differentiation of bowel loops and potential pathology; (b) One of the highest levels, 10. Presence of intra-abdominal fat provides contrast between bowel loops, despite the lack of enteric contrast.

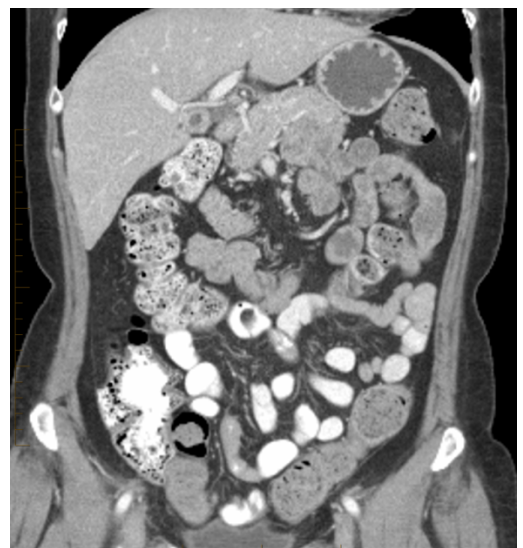

(a)

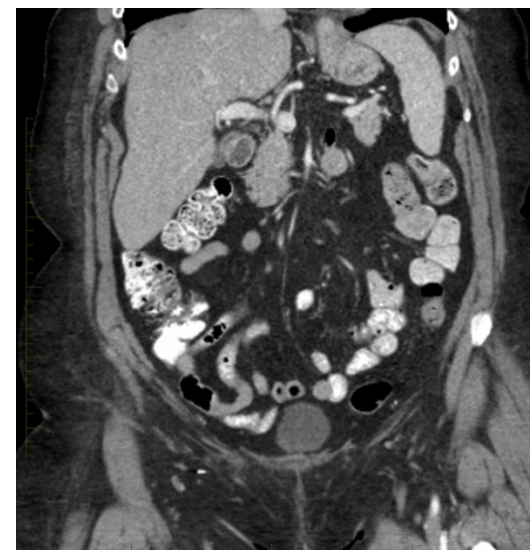

(b)

Figure 3. Coronal images with enteric contrast. (a) One of the lowest confidence levels, 6. Distribution of enteric contrast is limited to the distal small bowel and proximal large bowel; (b) One of the highest levels, 10. There is fairly uniform distribution of enteric contrast along with adequate intra-abdominal fat.

from the office or arrive early to their appointment. This additional step may adversely affect workflow in a busy outpatient office and can be a significant inconvenience for patients with limited mobility and access. A study in cancer patients reported that patients found that enteric contrast was the least pleasant experience, outranking intravenous cannulation and intravenous contrast injection [9]. Concern for perforated viscus is a concern specific to barium enteric contrast agents, but less of an issue in the ambulatory population. Another concern studied in the emergency setting includes prolonged gastric emptying, which can impact general anesthesia if an urgent surgical finding is identified.

The impact on the imaging center is increased cost. The list price of the enteric contrast agent used in this study (Readicat II ${ }^{\circledR}$ Barium Sulfate suspension $2.1 \% \mathrm{w} / \mathrm{v}, 2.0 \% \mathrm{w} / \mathrm{w}$ ) is $\$ 131.77$ for 24 bottles of the flavored variety and $\$ 135.12$ for 24 bottles of the unflavored variety. Less direct, but nonetheless important factors include additional staff necessary to appropriately screen and protocol cases for enteric contrast as well as distribute the contrast to the patient.

Positive enteric contrast may add to increased radiation exposure in the setting of automatic exposure control. A study comparing water with positive enteric contrast revealed an $11 \%$ increase in CTDIvol in patients who received positive enteric contrast (11.8 mGy versus $13.1 \mathrm{mGy}$, respectively) [11]. This benefit would need to be 
balanced with the potential risk of needing a repeat CT scan attributable to withholding enteric contrast. In our trial, no scan was repeated for this reason.

Limitations in our trial include a small sample size and the subjective analysis of confidence level by each interpreting radiologist. Recruitment was slower than expected. Of the 490 patients who scheduled CT examinations at eligible offices, 355 of them declined even initial contact with investigators. It is possible that this could have introduced selection bias into the study. Anecdotally, patients with a history of cancer were particularly concerned with whether an accurate comparison could be made with their prior study. Diagnostic accuracy would be an objective endpoint, but difficult when assessing multiple disease processes that are not necessarily pathologically proven. Radiologist bias may be a concern, since there is no way to blind radiologists to study technique. The comfort of radiologists in interpreting CT without enteric contrast is likely multifactorial including the study indication, practice setting and style and training.

\section{Conclusion}

Our findings indicate that the decision to routinely use enteric contrast may not be justified, given the lack of additional confidence it imparts. Further research is needed to evaluate the effect of enteric contrast on diagnostic accuracy in the outpatient setting.

\section{Acknowledgements}

We thank the CT and Call Center staff of Jefferson Radiology particularly Rebecca Rudnick as well as Luce Buhl and Crystal Kollmorgan.

\section{References}

[1] Laituri, C.A., Fraser, J.D., Aguayo, P., Fike, F.B., Garey, C.L., Sharp, S.W., Ostlie, D.J. and St Peter, S.D. (2011) The Lack of Efficacy for Enteric Contrast in the Diagnosis of Appendicitis by Computed Tomography. Journal of Surgical Research, 170, 100-103. http://dx.doi.org/10.1016/j.jss.2011.02.017

[2] Anderson, S.W., Rhea, J.T., Milch, H.N., Ozonoff, A., Lucey, B.C. and Soto, J.A. (2010) Influence of Body Habitus and Use of Enteric Contrast on Reader Confidence with Suspected Appendicitis Using 64 MDCT. Emergency Radiology, 17, 445-453. http://dx.doi.org/10.1007/s10140-010-0875-x

[3] Latifi, A., Labruto, F., Kaiser, S., Ullberg, U., Sundin, A. and Torkzad, M.R. (2011) Does Enteral Contrast Increase the Accuracy of Appendicitis Diagnosis? Radiologic Technology, 82, 294-299.

[4] Anderson, S.W., Soto, J.A., Lucey, B.C., Ozonoff, A., Jordan, J.D., Ratevosian, J., Ulrich, A.S., Rathlev, N.K., Mitchell, P.M., Rebholz, C., Feldman, J.A. and Rhea, J.T. (2009) Abdominal 64-MDCT for Suspected Appendicitis: The Use of Enteric and IV Contrast Material versus IV Contrast Material Only. AJR, 193, 1282-1288. http://dx.doi.org/10.2214/AJR.09.2336

[5] Holmes, J.F., Offerman, S.R., Chang, C.H., Randel, B.E., Hahn, D.D., Frankovsky, M.J. and Wisner, D.H. (2004) Performance of Helical Computed Tomography without Enteric Contrast for the Detection of Gastrointestinal Injuries. Annals of Emergency Medicine, 43, 120-128. http://dx.doi.org/10.1016/S0196-0644(03)00727-3

[6] Lane, M.J., Katz, D.S., Ross, B.A., Clautice-Engle, T.L., Mindelzum, R.E. and Jeffrey Jr., R.B. (1997) Unenhanced Helical CT for suspected Acute Appendicitis. AJR, 168, 405-409. http://dx.doi.org/10.2214/ajr.168.2.9016216

[7] Paulson, E.K. and Coursey, C.A. (2009) CT Protocols for Acute Appendicitis: Time for Change. AJR, 193, $1268-1271$. http://dx.doi.org/10.2214/AJR.09.3313

[8] Lee, S.Y., Coughlin, B., Wolfe, J.M., Polino, J., Blank, F.S. and Smithline, H.A. (2006) Prospective Comparison of Helical CT of the Abdomen and Pelvis without and with Enteric Contrast in Assessing Acute Abdominal Pain in Adult Emergency Department Patients. Emergency Radiology, 12, 150-157. http://dx.doi.org/10.1007/s10140-006-0474-Z

[9] Harieaswar, S., Rajesh, A., Griffin, Y., Tygi, R. and Morgan, B. (2009) Routine Use of Positive Enteric Contrast Material Is Not Required for Oncology Patients Undergoing Follow-Up Multidetector CT. Radiology, 250, 246-253. http://dx.doi.org/10.1148/radiol.2493080353

[10] Grainger, R.G. and Thomas, A.M.K. (1999) Textbook of Contrast Media. 2nd Edition, Isis Medical Media Ltd., Oxford, 135-148.

[11] Wang, Z.J., Chen, K.S., Gould, R., Coakley, F.V., Fu, Y. and Yeh, B.M. (2011) Positive Enteric Contrast Material for Abdominal and Pelvic CT with Automatic Exposure Control: What Is the Effect on Patient Radiation Exposure? European Journal of Radiology, 79, e58-62. http://dx.doi.org/10.1016/i.ejrad.2011.03.059 